\section{Cervicofacial Emphysema, Pneumomediastinum and Pneumothorax Caused by a Dental Procedure}

Sir,

Simple subcutaneous emphysema caused by dental intervention was initially described in 1900. Clinical studies that followed, attributed this pathologic condition to endodontic treatments, air-generating dental instrumentation used for dental extraction and/or restoration, as well as trauma from oral biopsy. We describe here an exceptional case of cervicofacial emphysema caused by a dental extraction, which extended into the parapharyngeal and retropharyngeal areas, the mediastinum, also causing a limited pneumothorax.1,2

A 17-year girl was admitted at the Emergency Department with mild pain and oedema over the left cheek, the left half of the neck, and the temporal region. She was referred by her dentist who had performed an extraction of her left third molar under local anaesthesia, using a standard high-speed-air-driven drill half an hour ago.

Physical examination revealed swelling and crepitus of the aforementioned areas. Her arterial pressure was 115/68 $\mathrm{mmHg}$, heart rate 76 beats/minute, and temperature within normal limits. Auscultation showed clear respirations bilaterally, phonation was normal, and visual acuity remained unchanged. Intraoral examination demonstrated no signs of infection or pathological dentition. Complete blood count, as well as hepatic and renal biochemistry, revealed normal values.

The patient was subjected to an emergency helical multi-sliced computerised tomography (CT) of the head, neck and thorax. The thoracic cavity was also scanned because the subcutaneous emphysema, which was clinically revealed, covered an extended area of the neck, triggering the suspicion of a possible pneumomediastinum. The CT revealed subcutaneous emphysema that extended from the buccal region to the submandibular, parapharyngeal areas, and the mediastinum. Furthermore, pneumomediastinum and a limited 2-3 cm upper pneumothorax bilaterally were shown (Figures $1 \mathrm{~A}$ and $1 \mathrm{~B}$ ). The CT scan depicted no fluid collection or neoplastic mass.

The girl was admitted for cardiopulmonary monitoring and prophylactic intravenous antibiotic treatment. The cervicofacial oedema was reduced and the patient was discharged the third day on oral antibiotics. She was

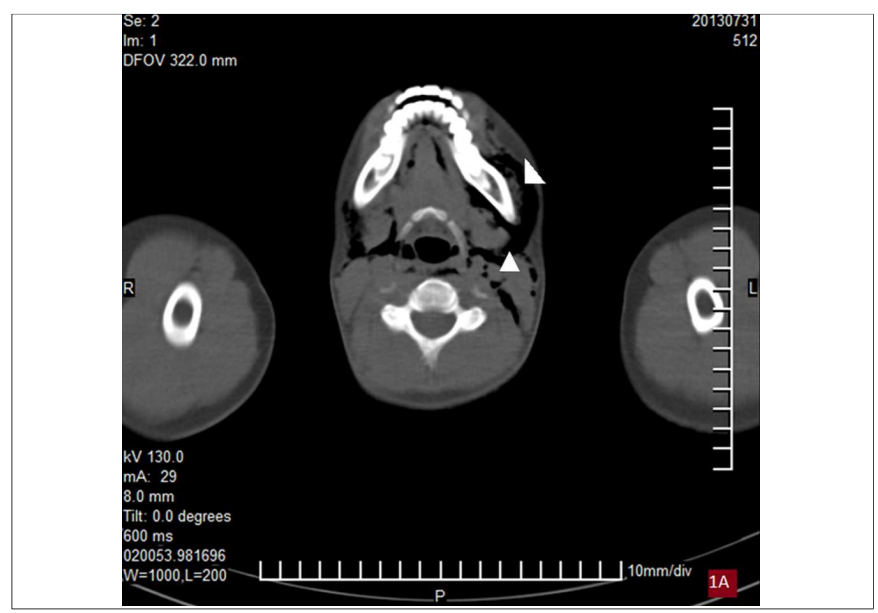

Figure 1A: Emergency helical multi-sliced computerised tomography of the head-neck of a 17-year-old girl, showing subcutaneous emphysema that extended from the buccal region to the left submandibular and parapharyngeal areas (white triangles), following the extraction of her left third molar by a dentist with a standard high-speed-air-driven drill.

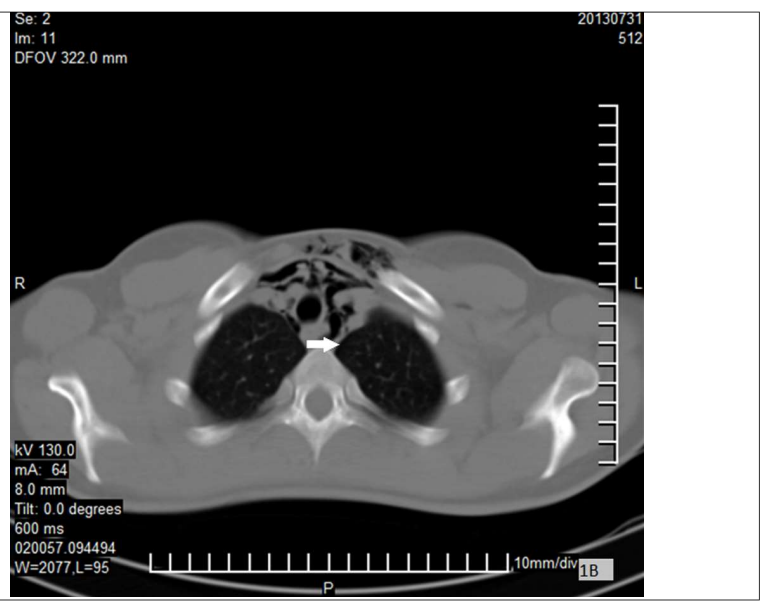

Figure 1B: Emergency helical multi-sliced computerised tomography of the thorax of a 17-year-old girl, showing pneumomediastinum and a limited 2-3 cm upper pneumothorax (white arrow), following the extraction of her left third molar by a dentist with a standard high-speed-air-driven drill.

reassessed with a new CT scan after a month, and no air in the mediastinum or the pleural cavity was demonstrated.

Most of the patients present a complete resolution of the pathology after maximum 7-10 days. However, rare life-threatening complications may occur, such as infective mediastinitis and sepsis due to oral cavity flora migration to the mediastinum, trachea compression, cardiac tamponade or air embolism. In general, treatment includes antibiotic prophylaxis and analgesics, if necessary.3-5 This patient was also conservatively treated, although she presented a pneumomediastinum and small pneumothorax, which are extremely infrequent. Undoubtedly, emergency physicians should be aware of this rare entity that occurs after dental procedures or else they may misdiagnose it as a haematoma, allergic reaction or infection. 


\section{REFERENCES}

1. Elia F, Laface B, Pagnozzi F, Boccuzzi A, Ferrari G, Perna M, et al. Cervicofacial emphysema and pneumomediastinum complicating a dental procedure. J Emerg Med 2013; 45:e179-81.

2. Mather AJ, Stoykewych AA, Curran JB. Cervicofacial and mediastinal emphysema complicating a dental procedure. J Can Dent Assoc 2006; 72:565-8.

3. Bocchialini G, Ambrosi S, Castellani A. Massive cervicothoracic subcutaneous emphysema and pneumomediastinum developing during a dental hygiene procedure. Case Rep Dent 2017; 2017:7016467.

4. Ramnarine $M$, Dubin Z. Cervicofacial and mediastinal emphysema due to a dental procedure. J Emerg Trauma Shock 2017; 10:34-6.
5. Nishimura T, Sawai T, Kadoi K, Yamada T, Yoshie N, Ueda T, et al. latrogenic subcutaneous emphysema and pneumomediastinum following a high-speed air drill dental treatment procedure. Acute Med Surg 2015; 2:253-6.

Konstantinos A. Paschos and Anestis Chatzigeorgiadis

Department of General Surgery, General Hospital of Drama, Drama, Greece

Correspondence: Dr. Konstantinos A. Paschos, Terma Ippokratous st, GR-66100, Drama, Greece

E-mail:kostaspaschos@yahoo.gr

Received: February 02, 2018; Accepted: October 23, 2018

…….... 